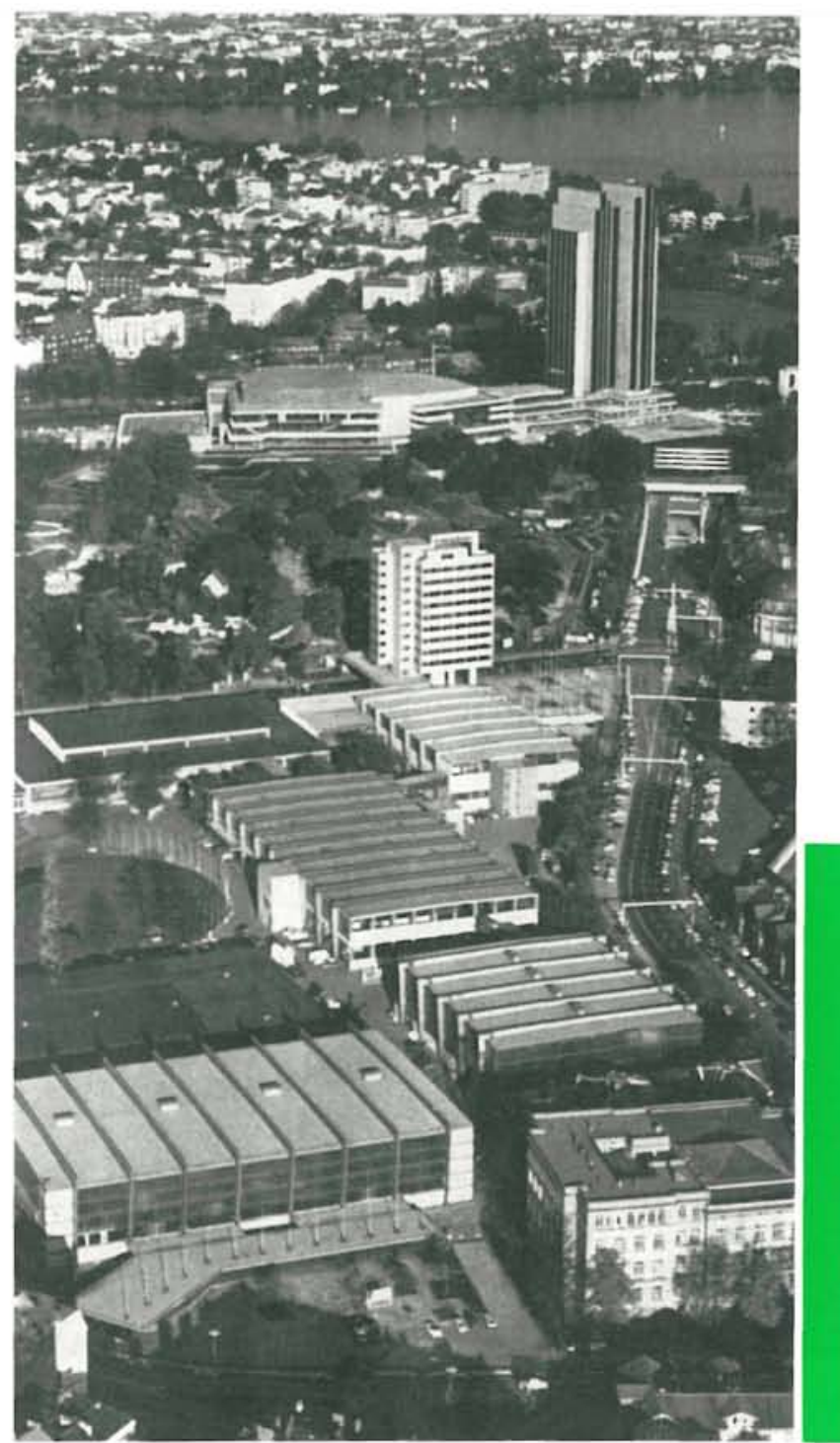

\title{
sinopsis
}

Levantada junto at jardin botánico de la ciudad, esta obra está constituida por el Centro de Congresos, propiamente dicho, y un gran Hotel, unido directamente al anterior.

El Centro se organiza de la siguiente manera:

Vestibulo de entrada que distribuye la circulación peatonal a: numerosas salas de distintos tamanos, locales de prensa, administracion, salas de banquetes y fiestas, zona de exposición y otras dependencias de la planta baja.

Primera planta con un restaurante y pequeñas salas de conferencias.

Segunda planta con dos grandes salas de 3.000 y 1.500 plazas, respectivamente: dispone de acondicionamiento acústico para posibilitar la celebración de conciertos.

$Y$, por último, dos sótanos de aparcamiento con una capacidad total de 1.100 vehículos.

El edificio del Hotel, por su parte, consta de una construcción alta, de 27 plantas, destinada totalmente a habitaciones, y otra baja, de 4 plantas. que contiene: la entrada con cafeteria, bar y restaurante; un primer nivel que dispone de piscina cubierta y sauna.

El conjunto cuenta con numerosos accesos en los que se ha evitado completamente el cruce de vehículos y peatones, mediante el empleo de rampas elevadas y pasajes subterráneos.

\section{centro de congresos en HAMBURGO ALEMANIA FEDERAL}

J. SCHRAMM Y G. PEMPELFORT, arquitectos BDA

$145-87$

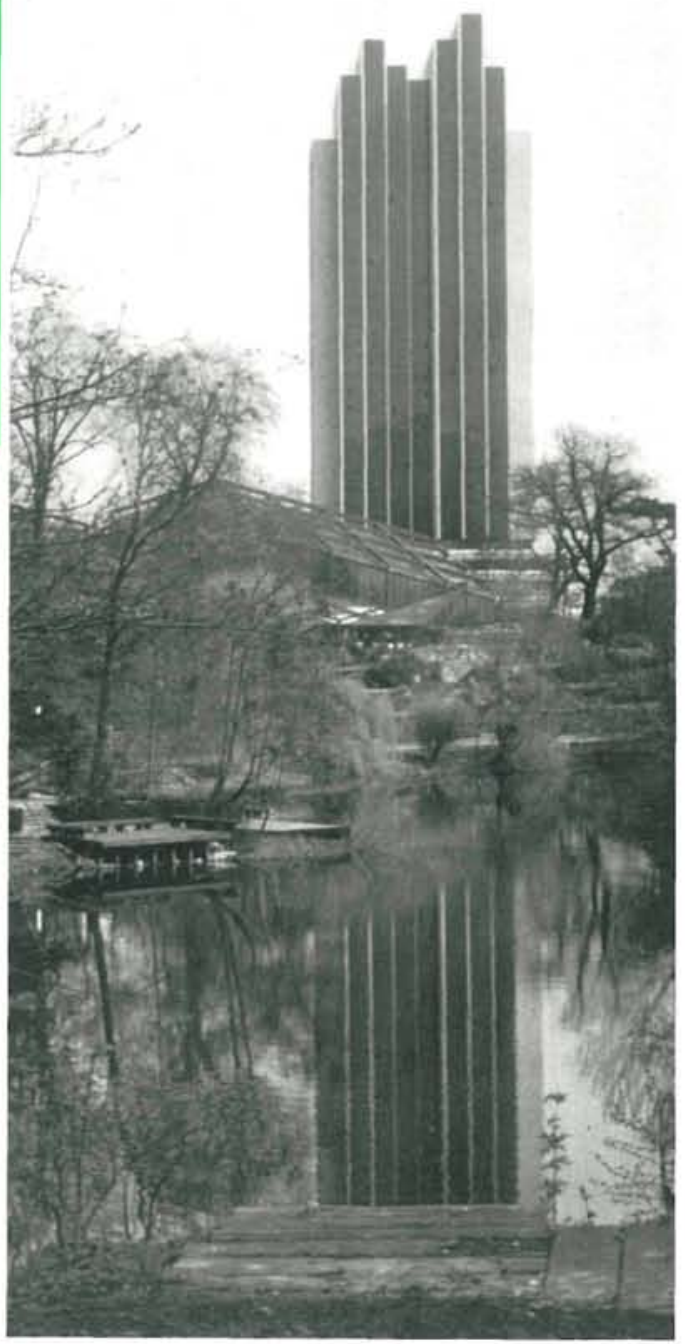



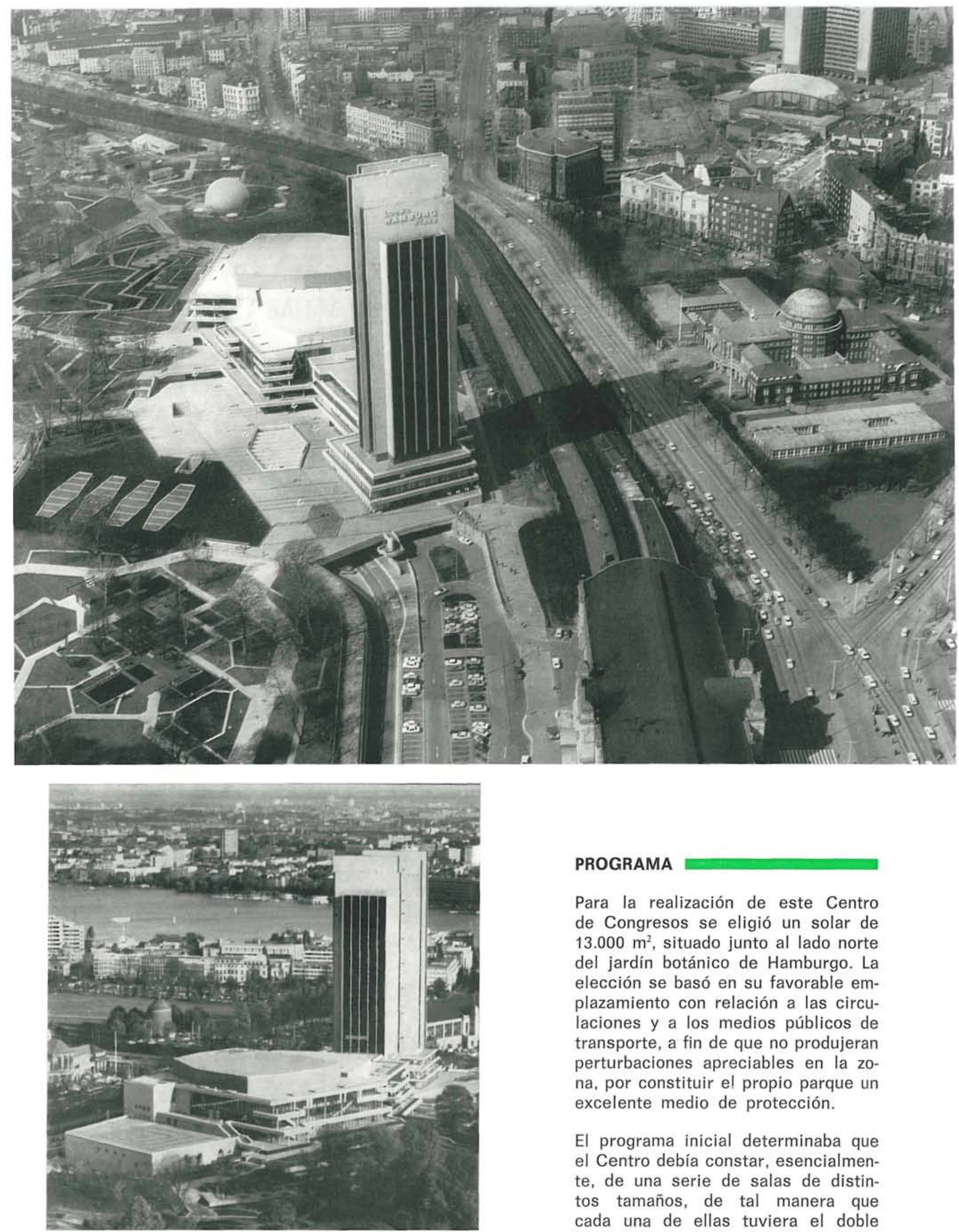

PROGRAMA

Para la realización de este Centro de Congresos se eligió un solar de $13.000 \mathrm{~m}^{2}$, situado junto al lado norte del jardín botánico de Hamburgo. La elección se basó en su favorable emplazamiento con relación a las circulaciones y a los medios públicos de transporte, a fin de que no produjeran perturbaciones apreciables en la zona, por constituir el propio parque un excelente medio de protección.

El programa inicial determinaba que el Centro debía constar, esencialmente, de una serie de salas de distintos tamaños, de tal manera que cada una de ellas tuviera el doble 


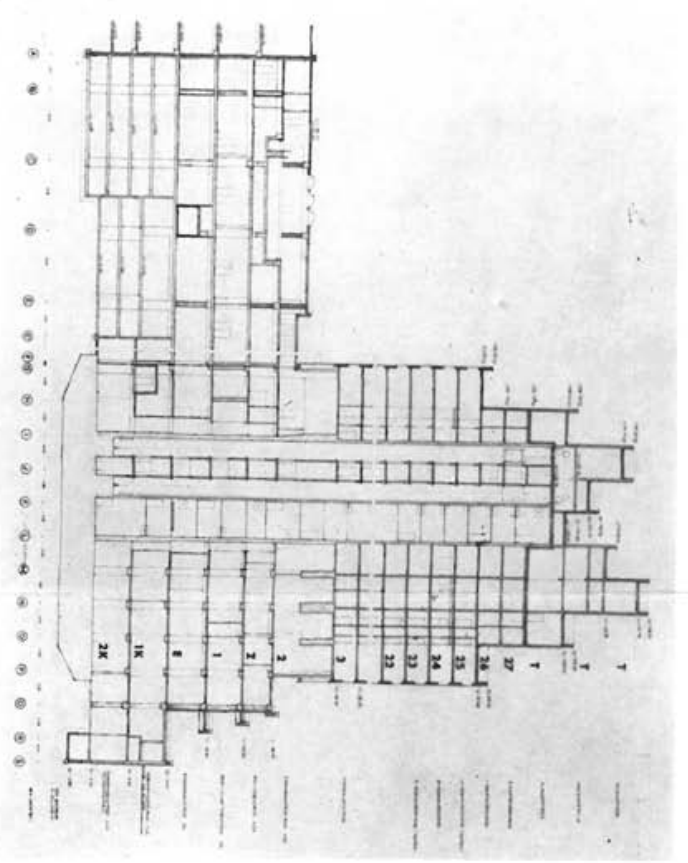

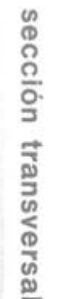
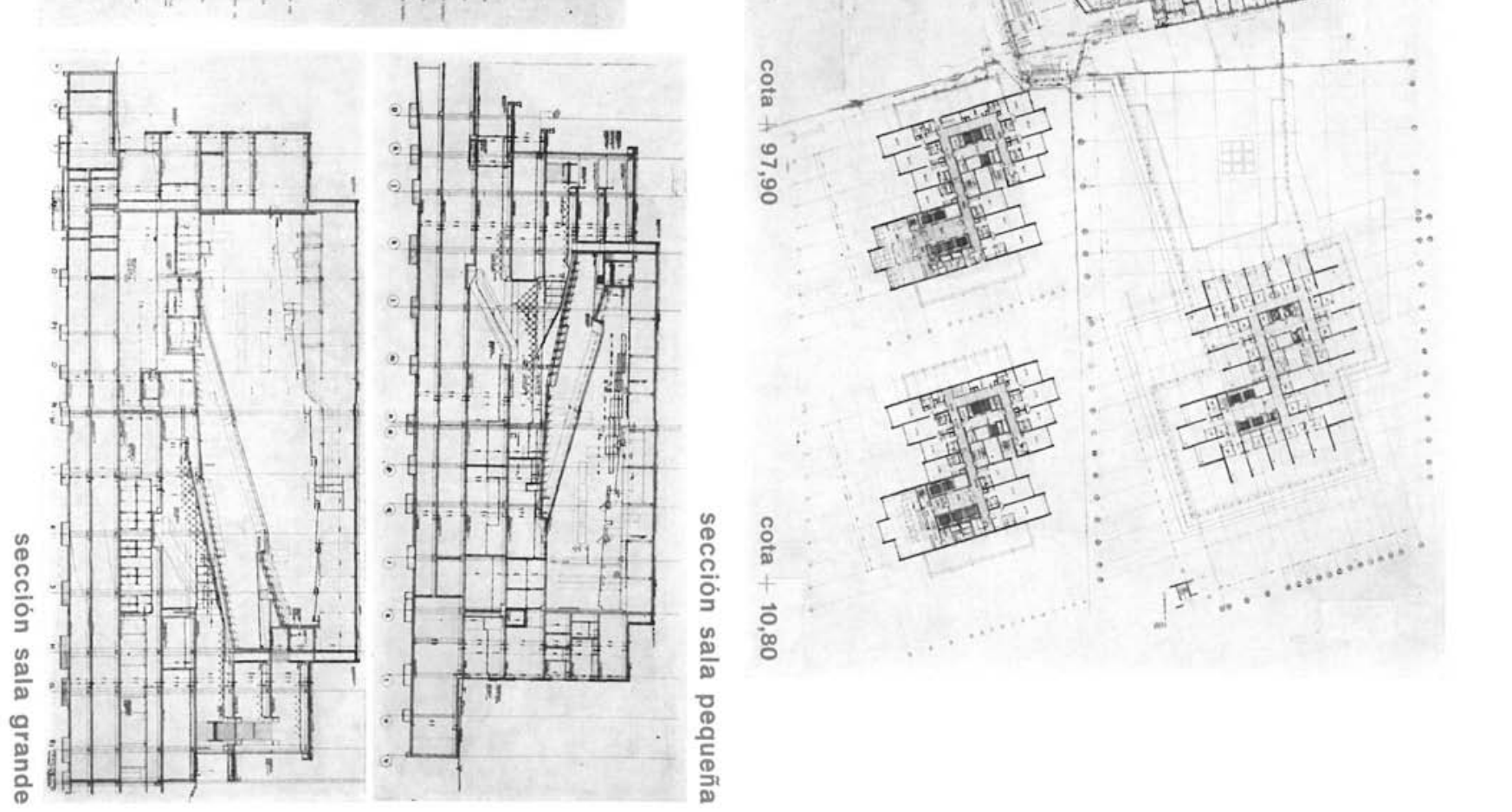

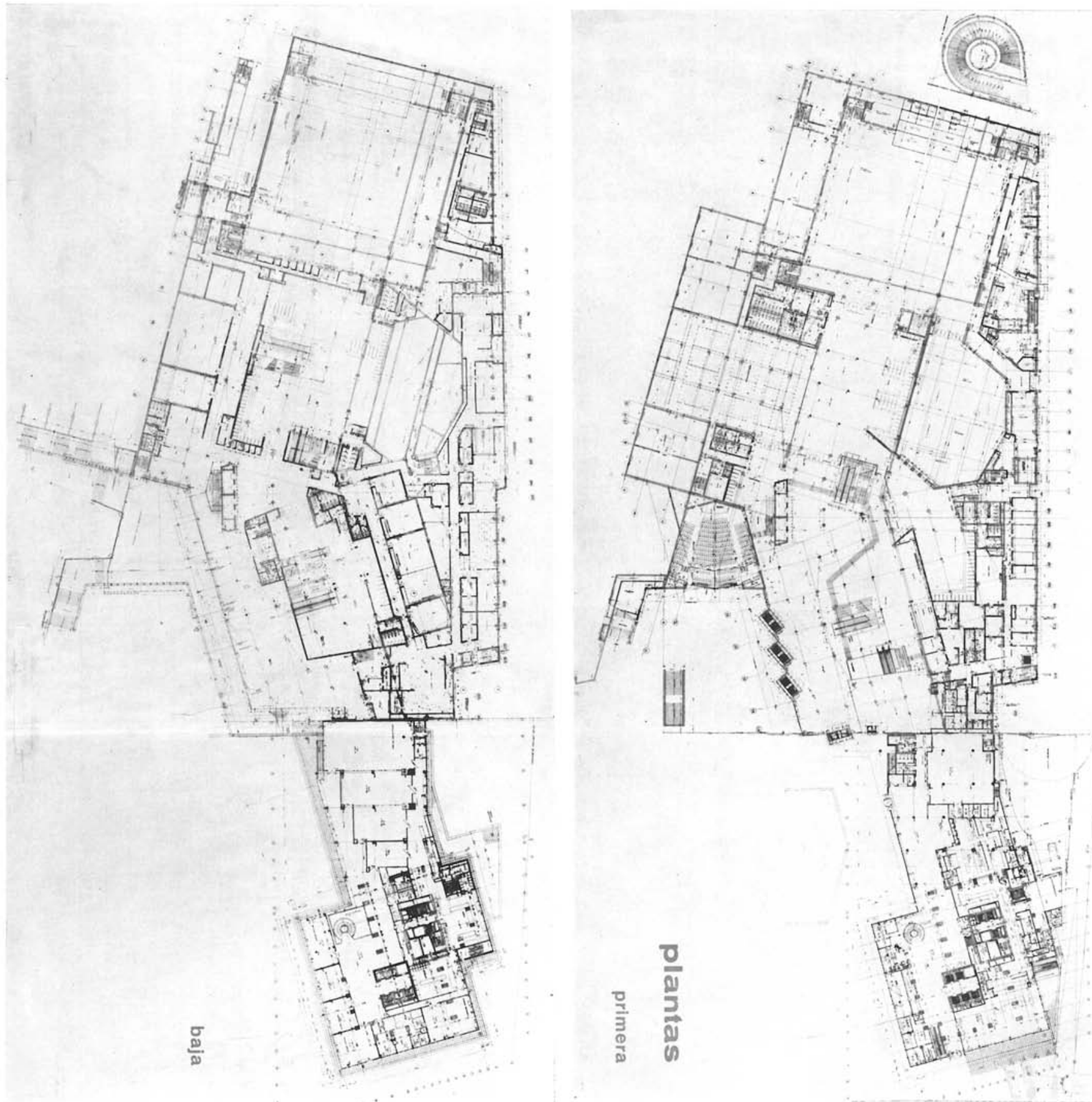
número de asientos que la inmediata inferior. En unión con el Centro. un hotel de gran rendimiento completaría el conjunto.

Se encargó a cuatro conocidos directores de congresos el dictamen pericial acerca del programa de locales, los cuales determinaron que la sala mayor debía tener 3.000 plazas. De aquí resultaron las salas siguientes de $1.500,750,375$, etc. asientos, decreciendo hasta las salas de coloquios de $30 \mathrm{~m}^{2}$. Se exigió, además, una sala de fiestas con suelo liso y mesas para banquetes, con capacidad de 1.500 personas, determinándose asimismo las necesidades de locales para la administración, prensa, gastronomía, etc.

Un comité formado por lós participantes estableció, después de numerosas inspecciones en centros de congresos extranjeros, los complementos necesarios del programa, eligiéndose el proyecto final entre las variantes presentadas.

Se tardó aproximadamente tres años en la construcción del edificio, desarrollándose paralelamente al mismo la realización del hotel.

\section{ACCESOS}

Entre los medios de comunicación la zona cuenta con una cercana estación de ferrocarril con paradas de tranvías, metro y autobuses, habiéndose dispuesto, además, en el solar, locales de espera para taxis y autobuses especiales.

Respecto a los vehículos privados se ha procurado, en todo momento, evitar cruces, para lo cual se bajó la calle adyacente a un plano subterráneo, ampliándola al mismo tiempo hasta formar una gran avenida. El acceso de los visitantes se efectúa por varios ramales que conducen a las dos plantas del garaje subterráneo, de 1.100 plazas de capacidad, de las cuales 200 pertenecen al hotel. Para casos de emergencia se ha previsto una salida directa desde los garajes a la avenida.

El conjunto de abastecimientos, así como la salida de desperdicios y los vehículos de transmisiones de televisión y radiotelefonía, tienen su llegada a los edificios a través de la misma avenida.

Los accesos peatonales, desde las vías públicas de comunicación hasta el Centro, se han trazado también sin cruces. Una gran plaza para peatones, creada delante del Centro, lo comunica directamente con el parque.

Una notable y acertada transformación del antiguo jardín botánico ha ofrecido la posibilidad de adaptar al entorno los nuevos edificios. De este modo surgió, delante de la construcción, una colina artificial bajo la que se halla enterrado parte del Centro, lo que ha permitido que las tres plantas superiores estén en contacto directo con el espacio circundante, pudiéndose salir al aire libre desde todas las salas. Afortunadamente se logró conservar los grupos principales de árboles, lo que, unido a la incorporación de grandes terrazas y nuevas plantaciones, ha contribuido a aumentar esa adaptación.

\section{EL EDIFICIO}

El atrio de entrada, dentro del edificio, asume la función de plataforma giratoria que distribuye a las salas las corrientes de peatones. Desde aquí se puede acceder a todos los 
guardarropas, directamente o por medio de escaleras mecánicas. También se encuentran, junto al atrio de entrada, las zonas de prestación de servicios, como: locales de prensa, administración, banco, correos y otras dependencias. El restaurante se aloja en el primer nivel, directamente accesible desde la entrada.

Todas las salas con suelo plano, adecuadas para banquetes o bailes, se hallan en la planta baja, pudiéndose llegar a ellas sin utilizar las escaleras. Igualmente, en dicha planta está la zona de exposiciones, que puede utilizarse para las que acompañan a los congresos o, subdividirse, a voluntad, con ayuda de un sistema de divisiones móviles. Así se pueden crear pequeños locales de oficinas, que en algunos congresos son precisas en gran número.

Las salas para 3.000 y 1.500 personas se encuentran en la segunda planta, llegándose a ellas por grupos de escaleras mecánicas. Orientadas hacia el parque y distribuidas en tres planos, se hallan las salas de estar. Los pequeños salones de conferencias están en el primer nivel, coordinados en dos grupos con las salas 1 y 2 , aunque pueden utilizarse también independientemente para pequeños congresos.

Para proporcionar a las salas 1 y 2 la posibilidad de organizar conciertos, tuvo que reducirse el nivel de perturbación. Para ello hubo que tenerse en cuenta las fuentes de ruido del solar: una calle principal muy recargada, los ferrocarriles federales, el tranvía con considerables ruidos de frenadas y sacudidas en el sector de la estación y, finalmente, las influencias del tráfico aéreo.

La pared exterior del sótano, que da a la estación, se reforzó, en $40 \mathrm{~cm}$, contra las sacudidas. Para reducir las perturbaciones producidas por el ruido del tráfico, las salas que dan al lado de la estación fueron rodeadas por dependencias secundarias. Contra el ruido de los vuelos aéreos se configuraron los techos por triplicado: un techo delimitador del espacio; el techo de sustentación, de piezas prefabricadas de hormigón pesado y, sobre el aislamiento contra el calor y la humedad, placas de $5 \mathrm{~cm}$ de espesor, colocadas sobre un lecho de gravilla de $5 \mathrm{~cm}$.

Especial cuidado se puso en la protección entre las salas superpuestas 1 y 4, para evitar las influencias perturbadoras, procedentes de los fosos de la orquesta, sobre la sala inferior. También aquí se dispuso un techo por triplicado y, por medio del aislamiento, se impidió que las piezas de hormigón de los fosos de la orquesta provocaran vibraciones.

Mientras que en la mayor parte de las salas hubo que cumplir ciertos requisitos acústicos, mediante la realización de paredes fono-absorbentes en los puntos requeridos, en el caso de la sala 1 , y debido a su tamaño poco habitual y también a su adaptación para conciertos, se presentaron considerables problemas. Para lograr una perfecta mezcla de las diferentes fuentes de sonido, el techo del local se equipó con superficies verticales de reflexión, paralelas al eje de la sala. Con objeto de conservar el lado inferior con la inclinación necesaria para la reflexión del sonido, el techo se desplegó, también verticalmente, al eje de la sala. Gracias a esto resultó un pronunciado escalonamiento del techo, que resulta cada vez mayor en dirección al estrado o escenario. El techo es plano por encima del estrado, disponiendo de las aberturas necesarias para las técnicas escénicas, que pueden cerrarse con trampillas en caso de conciertos. Delante de los muros de sus- 


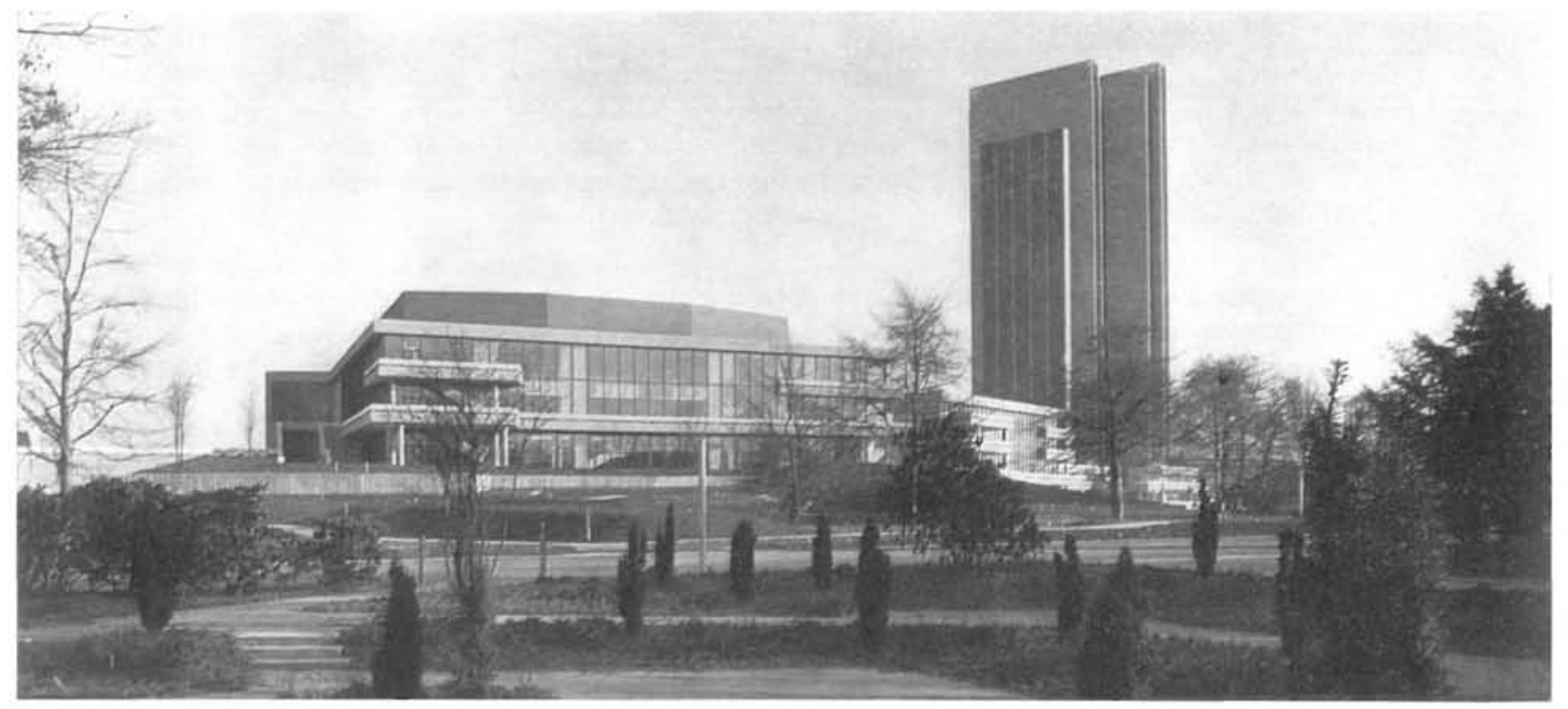

tentación se ha dispuesto una lámina de ladrillo rojo, que tiene una formación como de escamas, con caras paralelas al eje de la sala, estando sus superficies absorbentes atravesadas por ladrillos con orificios.

\section{CONSTRUCCION}

Gracias al buen terreno de fundación, la cimentación se realizó con facilidad. Las capas de agua existentes se derivan por medio de los correspondientes drenajes.

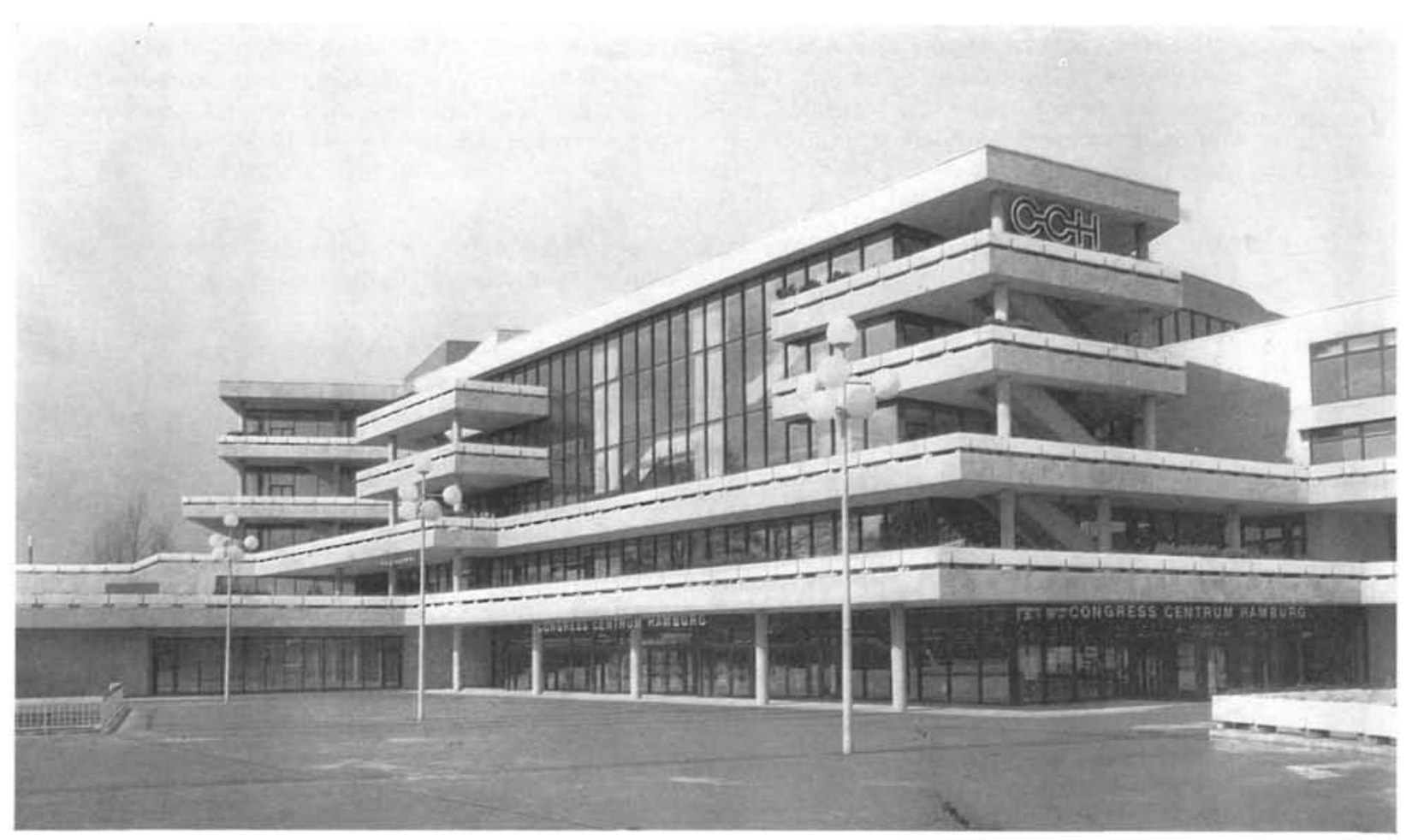


La estructura del Centro se realizó por medio de soportes y vigas de hormigón armado, con placas de arriostramiento. Las tres grandes salas están unidas con tirantes de acero, sobre los que se colocaron las losas de hormigón armado. Los anfiteatros de las salas 1 y 2, así como cada uno de los apuntalamientos, se realizaron con hormigón pretensado especial.

El revestimiento exterior del edificio está constituido por un chapado de roca conglomerada, que se aplicó sobre anclajes inoxidables. En los balcones se cclocaron placas de hormigón sobre cama de gravilla. Las jardineras son elementos prefabricados de hormigón blanco. Las ventanas y paneles de vidrio están fabricados a base de aluminio duro anodizado, de color bronce.

Todas las salas de la planta baja, adecuadas para bailes, así como los estrados, han sido provistos de un pavimento especial de gran dureza. Los locales de congresos, escaleras y salas de estar han sido equipados con alfombras, cuyos colores representan, al mismo tiempo, una guía óptica para las diferentes salas. Sólo la sala 1, por razones acústicas, tiene un suelo de linóleo. El atrio de entrada ha sido revestido de granito.

A excepción de la sala 1, cuyas paredes son de ladrillo, y de las salas 2 y 6 a 16 , que tienen paredes de madera decapada en color, sobre base incombustible, todos los demás locales públicos han sido revestidos de arpillera, tratada con pinturas de resina artificial.

Las salas 1, 2, 4 y 6 a 16 tienen techos Rabbitz por razones acústicas. En la sala 3 se ha dispuesto un techo de chapa perforada, con diferentes refuerzos, para configurarla como reflectante o como absorbente. La sala 5 -zona de conferencias y de exposiciones de utilización variable-, así como el atrio de entrada y todas las salas de estar han sido equipadas con techos de fibra mineral fono-absorbentes, tratados en parte con pintura.

En las salas 1 y 2 se han dispuesto asientos plegables, cuyas filas pares pueden transformarse en mesas, mediante el simple abatimiento de los respaldos. Estas salas pueden emplearse, por tanto, en una sesión parlamentaria, con la mitad de la capacidad habitual. Las demás salas tienen sillas móviles y mesas trapezoidales, rectangulares o redondas según las necesidades. En las salas de estar se emplean asientos ortogonales, que mediante la incorporación de elementos de mesa, pueden agruparse en la forma que se quiera.

Para su iluminación, el atrio de entrada y las escaleras están provistos de un sistema de lámparas incandescentes, que tiene la función de guía óptica de circulaciones.

La sala 1 puede iluminarse directa e indirectamente con lámparas fluorescentes incorporadas en la construcción, o con proyectores situados en el techo. Las paredes de ladrillo son iluminadas desde arriba con una iluminación marginal continua. Para el escenario se utilizan, desde arriba y desde los lados, además de los proyectores del techo, otros proyectores que confieren al escenario la claridad necesaria para las tomas de televisión en color. Todos los sistemas se pueden regular continuamente y mezclarse entre sí.

La sala 2 se pensó principalmente para congresos, por lo cual se ha provisto de una iluminación de lámparas fluorescentes. Las paredes cuentan con lámparas incandescentes e iluminación marginal.

En la sala de fiestas se ha dispuesto un sistema de lámparas de más de 1.200 cilindros de aluminio, con luces que brillan alternativamente, incluyendo, además, luces de colores con las que se consiguen variados efectos. 
Para su fácil mantenimiento y reposición, las lámparas están suspendidas de un reticulado de perfiles en $U$ y conectadas a cajas de enchufes. En los puntos de entrecruzamiento del reticulado se han colocado dispositivos para poder suspender puentes adicionales, plataformas de cámaras, decoraciones, etc.

Todo el edificio ha sido eauipado con instalaciones de aire acondicionado.

Como medida de protección contra incendios, todos los locales están dotados con una instalación de rociadores automáticos.

\section{INSTALACIONES ESPECIALES}

El restaurante está subdividido en uno pequeño de 120 asientos, con servicio de camareros y, otro mayor, de 180 plazas con autoservicio. Delante hay una terraza al aire libre orientada hacia el parque En la entrada se ha dispuesto un bar con 40 asientos, como punto de encuentro para todo el conjunto.

Las cocinas están formadas por: una de producción; una para banquetes en las salas 3 y 4; otra para la galería de la sala 3; una estación de reparto para la sala 5 , y una cocina, a la carta, para los restaurantes. El lavadero está dispuesto en forma centralizada para todos los sectores.

En el lado norte del edificio se encuentran: los locales de administración, los talleres y almacenes, los guardarropas aislados y colectivos de los escenarios oficinas de alquiler y locales para la prensa - local de conferencias, de interviús, teléfonos y teletipos-. Además, se han añadido locales para multicopista, información y las dependencias médicas. En el sótano se han dispuesto guardarropas para el personal de servicio.

En las salas 1 a 4 se han montado cabinas de traducción simultánea para cinco idiomas. En los demás locales se pueden instalar cabinas desmontables. Cuenta el Centro con una instalación de televisión, en circuito cerrado, para la retransmisión de los congresos.

\section{HOTEL}

El Hotel se halla unido directamente al Centro de Congresos. Consta de un bloque alto de habitaciones, en forma de torre de 27 plantas, y de una construcción baja de cuatro, que contiene las instalaciones de funcionamiento y servicios.

Para eliminar asientos diferenciales se dispuso una losa de cimentación de 4 m de espesor, que permite el requerido desplazamiento de cargas equilibradas. En los bordes el espesor de la losa es de $2 \mathrm{~m}$. La construcción baja, separada del edificio alto por una junta de dilatación, está cimentada sobre puntos aislados o sobre zanjas.

La estructura del bloque alto es de pilares y vigas, mientras que en el bloque de dormitorios se utilizó una construcción a base de muros pantallas.

El desplazamiento de las cargas, desde el bloque de dormitorios hasta los soportes de la base, se efectúa mediante vigas de $4 \mathrm{~m}$ de canto.

La torre está recorrida en toda su altura por dos núcleos, en los que además de los as. censores se han alojado las cajas de las escaleras de emergencia y todas las líneas y conducciones de la instalación. 

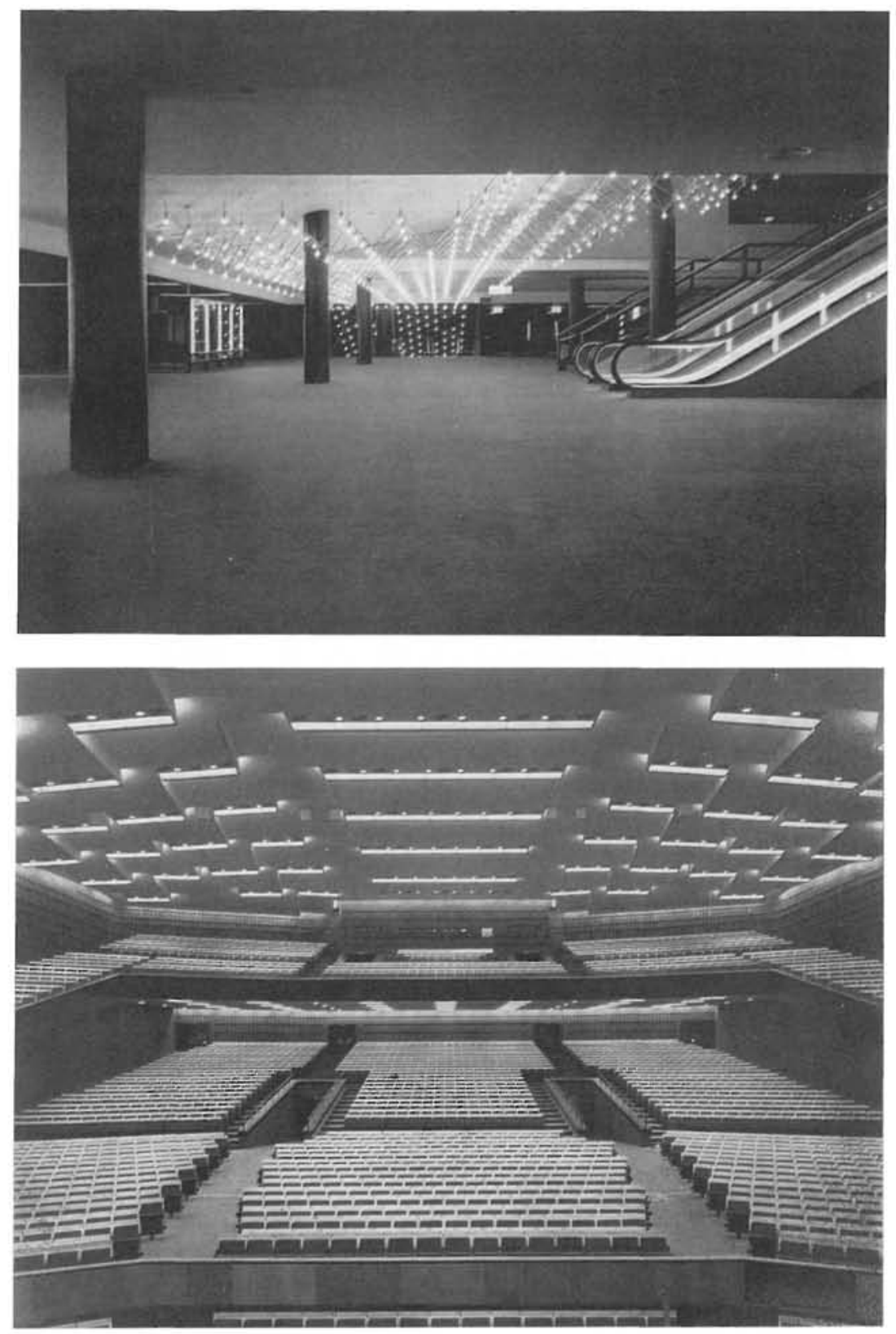

Fotos: JOHN WYAIT. Hanseatische Lufftote, Fürstenberg, Neur Haimat Stâdtobou

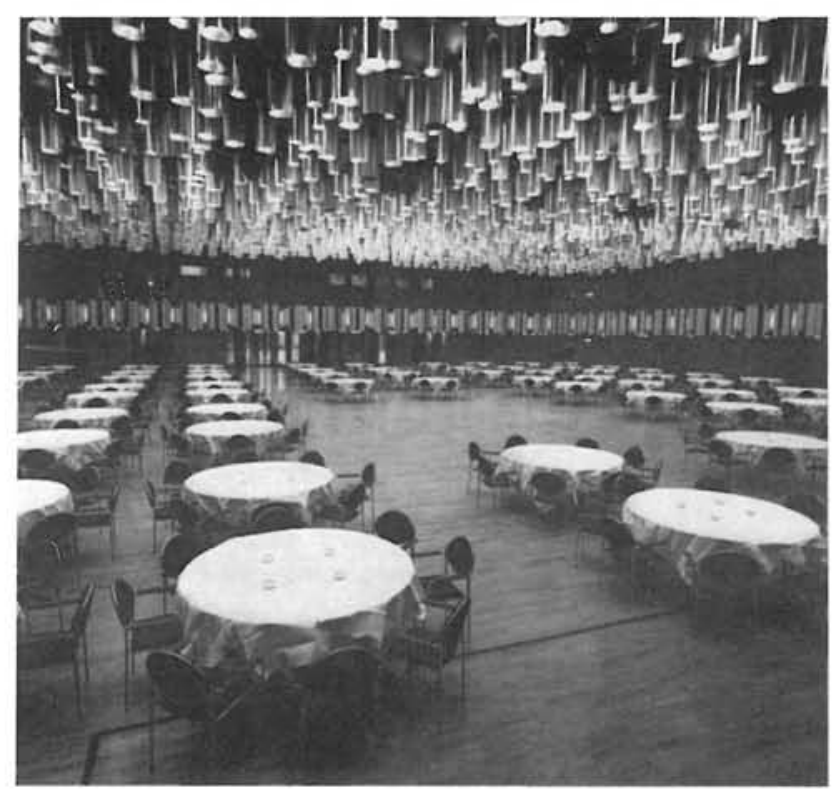

\section{interiores}

Las tres plantas técnicas, situadas en la parte superior de la torre, están separadas, por razones de insonorización, de la zona de dormitorios, por una junta horizontal continua.

Para la fachada se ha utilizado, en la parte frontal de las habitaciones, un muro cortina de aluminio, con perfiles de aluminio anodizado en color bronce. Las ventanas han sido acristaladas con vidrio grueso, por razones acústicas. Los balcones, al igual que los mamparos de la torre, están chapados con roca conglomerada

La climatización de los edificios bajo y alto se lleva a cabo de manera separada, mediante las dos centrales situadas en el sótano y en la parte alta de la torre, respectivamente.

\section{ORGANIZACION}

El sector de entrada tiene dos plantas y así, mientras los huéspedes llegan desde la plaza al hotel, a la altura de la planta noble, y por medio de escaleras mecánicas son llevados a la recepción de la planta baja, el peatón que llega desde la explanada del parque, accede directamente al atrio de entrada. 
Desde este último el huésped puede alcanzar inmediatamente la cafetería, el bar y el restaurante de especialidades. En este nivel existe una unión con la sala de estar del edificio de congresos.

Una escalera al aire libre conduce desde el atrio de entrada a los salones de estar, a la sala de baile y a los locales de conferencias de la primera planta, que también está unida directamente al edificio de congresos.

En la cubierta del edificio bajo se ha dispuesto una piscina cubierta, una sauna y todas las dependencias auxiliares necesarias.

Las 24 plantas de dormitorios están distribuidas en torno a dos núcleos de circulación, que quedan alternados entre sí (uno para huéspedes y otro para servicios). El primero alberga cinco ascensores y la caja de escaleras de emergencia, y el segundo, tres ascensores, dos cajas de escaleras y los montacargas.

Todas las unidades de habitación son del mismo tamaño, constando de un salón de estardormitorio, de $19 \mathrm{~m}^{2}$, y un cuarto de baño, de $4 \mathrm{~m}^{2}$. Cada habitación está climatizada individualmente, con un aparato combinado de calefacción-refrigeración, fijado sobre el techo descolgado del vestíbulo delantero; de esta manera, cada cliente tiene la posibilidad de regular a su gusto la temperatura ambiente de su habitación.

En las plantas 23 y 25 se han agrupado cuatro unidades de habitación para formar suites.

En las dos plantas de sótano se han distribuido las centrales técnicas. almacenes de aprovisionamiento y garajes.

Los demás locales de servicios, como: guardarropa del personal, cafetería contabilidad, central telefónica, etc., se encuentran en la segunda planta, entre la de los salones de conferencias y la de la piscina.

\section{résumé}

Centre de Congrès à Hambourg. République fédérale d'Allemagne

J. Schramm et G. Pempelfort,
architectes BDA

Bâti près du jardin botanique de la ville, cet ouvrage est constitué par le Centre de Congrès, proprement dit, et par un grand hôtel, uni directement à ce Centre. Le Centre de Congrès est organisé de la manière suivante:

- Vestibule d'entrée qui distribue la circu. lation vers les nombreuses salles, les ministration, les salles pour les banquets, la zone d'exposition et d'autres locaux du rez-de-chaussée.

- Premier étage occupé par un restaurant et de petites salles de conférences.

- Deuxième étage réservé à deux grandes salles de 3.000 et 1.500 places, respectiement. Il a été conditionné acoustiquement pour tionnement, de 1.100 véhicules.

Le corps de I'hôtel, pour sa part, comprend un bâtiment haut, de 27 étages, destine entièrement à des chambres, et un autre plus bas, de étages, ou se trouvent de séjour, le bal et les locaux de confé. rences, en plus de la piscine couverte et du sauna.

L'ensembe dispose de nombreux accès, où le croisement de véhicules et piétons a été évité par l'aménagement de rampes sur

\section{summary}

Congres Centre in Hamburg. Federal Germany

J. Schramm and G. Pempelfort, architects BDA

Built near the town's botanical garden, this work is made up of the Congress Centre, as such, and The Centre is orga

nized as follows:

- Entrance hall which distributes visitors to: numerous rooms of differing sizes. press rooms, management, banquet and dance halls, exhibition area and other premises on the ground floo

- First floor with a restaurant and small conference rooms.

Second floor with two large halls, seating 3,000 and 1,500, respectively; acoustic arrangements have been made so that
concerts can be held.

with space for 1,100 vehicles in all.

The Hotel building, by itself, has a tall part, with 27 storeys, designed entirely for rooms, and another low part, with 4 hoors, bar and restaurant; a first level with living room, dance hall and conference rooms: and another level with a covered swimming pool and sauna baths.

The whole unit has numerous entrances, in which the crossing of vehicles and pedes trians has been completely avoided, by using elev.

\section{zusammenfassung}

Kongresszenter in Hamburg. West Deutschland J. Schramm und G. Pempelfort, Architekten BDA

Dicht am Botanischen Garten der Stadt erhebt sich dieses Kongresszenter, das aus der eigentlichen Kongresshalle und einem grossen, Das Zenter ist folgender massen eingeteilt: vingangshalle, von der mehrere Säale waltungsräume, Fest. und Ausstellungssäale und andere Räume des Erdgeschos. ses ausgehen.

- Im ersten Stock befinden sich ein Restaurant und kleine Konferenzzimmer.

- Der zweite Stock weist zwei gross Sảale mit je 3000 und 500 Plätzen auf. Diese verfugen uber akustische Anlagen, Konzerte zu geben.

- Zuletzt zwei Parkkeller, die zusammen 1100 Wagen beherbergen können. Das Hotel besteht seinerseits aus einen Gebäude mit 27 Etagen, die nur für Zim. mer bestimmt sind, und einem niedrigeren Gebaude mit 4 Stockwerken, in welchem Rich der Eingang, die Cafeteria, Bar und fügt üher einen Aufenthaltsraum, Tanz ver. Konferenzsäale. In einem anderen Stock findet man ein überdachtes Schwimmbad und eine Sauna.

Die vielen Eingänge des Zenters vermeiden an Hand von erhöhten Rampen und Fussgän gertunneln die Kreuzung von Fahrzeugen und 